\title{
Motherhood and Empathy: Increased Activation in Empathy Areas in Response to Other's in Pain
}

Irene Sophia Plank ( $\square$ irene-sophia.plank@charite.de)

Charité - University Medicine Berlin

\section{Catherine Hindi Attar}

Charité - University Medicine Berlin

\section{Stefanie Kunas}

Charité - University Medicine Berlin

\section{Isabel Dziobek}

Humboldt-Universität zu Berlin

\section{Felix Bermpohl}

Charité - University Medicine Berlin

\section{Research Article}

Keywords: $\mathrm{fMRI}$, empathy, pain, motherhood, children

Posted Date: May 5th, 2021

DOI: https://doi.org/10.21203/rs.3.rs-478801/v1

License: (c) (i) This work is licensed under a Creative Commons Attribution 4.0 International License. Read Full License 
Motherhood and empathy: increased activation in empathy areas in response to other's in pain

\author{
Irene Sophia Plank ${ }^{1,2,3,4}$, Catherine Hindi Attar ${ }^{1}$, Stefanie Kunas ${ }^{1}$, \\ Isabel Dziobek ${ }^{2,3,4}$, Felix Bermpohl ${ }^{1,2,3}$
}

\begin{abstract}
${ }^{1}$ Department of Psychiatry and Psychotherapy, Charité - Universitätsmedizin Berlin, corporate member of Freie Universität Berlin and Humboldt-Universität zu Berlin, Berlin, Germany

${ }^{2}$ Einstein Center for Neurosciences, Charité - Universitätsmedizin Berlin, corporate member of Freie Universität Berlin and Humboldt-Universität zu Berlin, Berlin, Germany ${ }^{3}$ Berlin School of Mind and Brain, Humboldt-Universität zu Berlin, Berlin, Germany ${ }^{4}$ Department of Psychology, Institute of Life Sciences, Humboldt-Universität zu Berlin, Berlin, Germany
\end{abstract}

Corresponding author: Irene Sophia Plank; Luisenstr. 56, 10117 Berlin, Germany; phone: (030) 2093-89776; fax: (030) 2093-89751
Abstract:
183 words
Intro:
1,102 words
Methods:
1,617 words
Results:
1,385 words
Discussion:
1,609 words
Total (exc. Methods): 4,096 words (goal: max. 4,500 words) 


\begin{abstract}
Empathy as the capacity to feel with others is a vital human response. It is especially important in the context of parenting. To date, little is known about differences in empathy responses of parents and non-parents to children and adults. Therefore, in this study functional magnetic resonance imaging was used to shed further light on the interaction of motherhood and behavioural as well as neural responses to stimuli depicting adults and children in pain. Despite similar ratings on the imagined painfulness of the stimuli, mothers showed higher activation in the bilateral anterior insula, key regions of empathy for pain. Additionally, mothers increasingly activated other areas associated with social affect and cognition, including the inferior frontal gyrus, superior temporal gyrus and the medial superior frontal gyrus. Differences between adult and child stimuli were only found in occipital areas. This suggests that mothers have a stronger empathy response than non-mothers regardless of whether the target of their empathy is a child or an adult. This generalised difference in empathy indicates that motherhood might influence overall affective social understanding rather than specifically shape child-related empathy responses.
\end{abstract}

Keywords: fMRI $\cdot$ empathy $\cdot$ pain $\cdot$ motherhood $\cdot$ children 


\section{Introduction}

Humans' emotional capacity for empathy is vital to a social society and prosocial behaviour [1]. Empathy is an emotional response to other's emotions which is isomorphic in nature. Additionally, the person feeling empathy is aware of the other person being the source of the emotion [2]. Therefore, empathy is an important part of understanding others on an emotional level and part of the affective route of social understanding. In this usage, it is differentiated from cognitive social understanding which refers to inferential processes to understand other's emotions and mental states. Competence in cognitive and affective social understanding is largely independent of one another [3, 4]. However, it is important to note that both cognitive and social understanding are not mutually exclusive and work together in most situations [5]. Evolutionary perspectives on empathy propose that a general capacity for empathy developed out of parenting due to the increased duration of dependency in humans [6]. This perspective is corroborated by studies showing a positive effect of empathic and compassionate parenting on one's offspring. Parental trait empathy is positively related to a child's attachment security [7] and parents serve as important social models for children teaching them social competencies [8, 9].

Parenting by nature forces people to use cognitive and affective social understanding constantly when interacting with their child. When asked to describe infants' behaviour in a video, parents are more likely to describe mental states compared to non-parents [10]. Comparing valence ratings for infant faces between parents and non-parents reveals that mothers specifically tend to give more extreme ratings while this is not true for fathers [11]. This effect might influence a corresponding empathy response in mothers. In an attentional capture paradigm, infant faces led to slower response times than adult faces in women and this was more pronounced in mothers compared to non-mothers [12]. These findings were replicated and extended to pre-adolescent faces in distress [13]. In sum, these studies show that parents react differently to infants and children than non-parents.

Differences in social understanding due to parenthood also extend to the neural level. 
Using electroencephalographic (EEG) measures, Proverbio and colleagues reported higher sensitivity for differences in facial affect of infants in parents as reflected in the N2 [14]. Interestingly, differences in electrophysiological correlates of emotional face processing between parents and non-parents may extend to adult stimuli [15]. However, using an emotion recognition task and functional near-infrared spectroscopy (fNIRS), Nishitani and colleagues observed increased activity in the right prefrontal cortex in mothers compared to non-mothers in response to infant but not adult faces [16]. Parsons et al. used functional magnetic resonance imaging (fMRI) to compare mothers and non-mothers responses to adults' or children's distressed or neutral sounds [17]. Pooling distressed and neutral sounds, they showed that infant stimuli led to stronger activation in multiple areas, including the left amygdala and orbitofrontal cortex. Adult stimuli in turn activated the left middle frontal gyrus more strongly than infant stimuli. Contrasting mothers with non-mothers revealed higher activation for mothers in several areas, including the right middle frontal gyrus, precuneus, middle temporal gyrus as well as left superior temporal pole and the orbitofrontal cortex. Zhang and colleagues revealed increased activity in mothers in several frontal and occipital areas compared to non-mothers when viewing infant faces [18]. In sum, these studies showed neural differences between parents and non-parents in several aspects of social understanding, however, no study to date has investigated neural empathy and parenthood.

Thus, despite the lack of research on the interaction of empathy and parenthood, previous studies have investigated many related aspects of both the affective and cognitive route of social understanding. Taken together, research has shown that parenthood can have effects both on a behavioural and on a neural level even when unknown adults or children were the target [16, 12, 17, 15, 18]. Some studies additionally suggested that there is an interaction between parenthood and processing adult and child stimuli [16, 12, 17] while others hinted that differences apply to both adults and children [15]. It is still unclear whether the influence of parenthood extends to empathy and in what way the empathy response in parents and non-parents is different when reacting to children as compared to adults. 
Research on empathy responses in the brain has shown that observing other's in pain and feeling pain yourself leads to considerable overlap in activation [19]. Empathy for pain has proven to be highly open to influences dependent on the person experiencing empathy [20] and the person inducing empathy [21, 22]. This makes empathy for pain a well suited paradigm to investigate nuances in neural empathy responses like differences due to motherhood or comparing child to adult stimuli. For this study, we adapted a paradigm used by Lamm and colleagues to compare two groups of people inducing empathy [23], children and adults respectively. Another advantage of the empathy for pain paradigm is the existence of meta-analyses providing guidance for specific hypotheses regarding regions of interest. A recent meta-analysis examined different aspects of empathy and revealed four core regions for empathy for pain: the bilateral anterior insula (AI), the medial superior frontal gyrus (SFG) and the left supramarginal gyrus (SMG) [24]. Especially the bilateral AI are considered core regions of empathy for pain and meta-analyses consistently confirm their association with empathy for pain [25, 26]. This provides clear predictions as to which regions are expected to reflect possible modulations of empathy for pain responses.

This study aimed to address the influence of motherhood of the perceiver and age of the protagonist (adults and children) of the stimuli on empathy for pain as well as the interaction of both factors. The sample focussed on mothers because they provide primary care for children in most cases in Germany [27, 28]. Additionally, although previous studies reported vast overlaps and similarities in fathers and mothers, they also indicated possible differences that justify separate investigation [29, 11]. Specifically, this study aimed to answer the following questions: First, does motherhood interact with the empathy response of women? Second, are empathy responses larger when the source of the empathy is a child rather than another adult? Third, do mothers relative to nonmothers have stronger empathy responses to children? To answer these questions, we measured neural empathy responses to children and adults in mothers and non-mothers. We define empathy response as the difference between painful and neutral stimuli. We hypothesised (1) that mothers show stronger empathy responses to people in pain in 
general, (2) that children compared to adults in pain lead to higher empathy responses across groups and (3) that the increase of empathy response to children is even more pronounced in mothers.

\section{Methods}

\subsection{Participants}

Women were recruited online and with flyers and had to fulfil the inclusion criteria of being healthy, right-handed, cisgender female, between 25 and 50 years old and having sufficient knowledge of German. Mothers had at least one biological child between 4 and 10 years of age while non-mothers did not spend a significant amount of time with children (neither professionally nor in their personal life). Based on power analyses for a within-between interaction in a repeated-measures ANOVA with two groups and two measurements using $\mathrm{G}^{*}$ Power $(f=0.25, \alpha=0.05,(1-\beta)=0.95$, corr $=0.5)$ [30], we aimed for a sample size of 54 women (27 mothers). We excluded two participants due to artefacts and two because they rated both painful and neutral stimuli as not painful. Due to the outbreak of Covid-19, we did not replace them. The final analysed sample consisted of 50 women $(25$ mothers, mean age mothers $=38.28$; non-mothers $=35.64)$ and post-hoc power calculations indicated a power of 0.93 for a within-between interaction in a repeated-measures ANOVA in $\mathrm{G}^{*}$ Power $3(f=0.25, \alpha=0.05, n=50$, corr $=0.5)$. Mothers and non-mothers did not differ in age, intelligence or socio-economic status (see table 1). The study was approved by the Ethics committee of the Charité Universitätsmedizin Berlin and was conducted in accordance with the Code of Ethics of the World Medical Association (Declaration of Helsinki). All women received payment and gave written informed consent before participating.

\section{$2.2 \quad$ Experimental procedure}

Prior to scanning, participants read the study information with the consent form and had the opportunity to ask questions. Then, one of the experimenters conducted a semi- 
structured interview with the participants about their demographics, health, mood state, family and relationship to children. After the interview, participants performed a short IQ screening [31] followed by questionnaires to measure emotion regulation (ERQ [32]), relationship attachment (ECR-RS [33]), social desirability (KSE-G [34]), alexithymia (TAS-20 [35]) and trait empathy (IRI [36]). The empathy for pain fMRI task was presented in two runs of $8.5 \mathrm{~min}$. Additionally, participants performed another task in the scanner as well as a run of resting state fMRI. Scanning lasted about 60min and total experiment duration was about $90 \mathrm{~min}$.

\section{3 fMRI paradigm}

Participants viewed colour pictures of adults and children in painful and neutral situations. Each scenario was shown in four variations: painful for a child, neutral for a child, painful for an adult and neutral for an adult (see fig. 1A). Neutral pictures also contained the cause for the pain but without showing the actual painful situation. Empathy response was defined as the difference in neural responses associated with painful and neutral pictures. In total, 31 scenarios were created and photographed. The pictures were assessed by 22 subjects for imagined painfulness and matching of the scenarios. We chose 24 scenarios that were rated as well-matched and had a difference between painful and neutral scenarios of at least 30 on a scale from 0 to 100. This resulted in 24 stimuli per condition and 96 stimuli in total. There was no difference in rating between the child and adult stimuli. All pictures were matched in colour, luminance and contrast. Four neutral faces were chosen to introduce the scenarios and emphasised the protagonist of the following scenarios (female and male adult from the KDEF set [37], 4-year-old female and 5-year old male child from the CAFE set [38, 39]).

Each trial started with a face presented for 3s followed by four pictures of situations, two painful and two neutral, presented for $3.5 \mathrm{~s}$ each (see fig. 1B). The order of these four pictures was randomised. The participants were asked to imagine that the person whose face they saw was the protagonist of the displayed situations and to put themselves in their shoes. Pictures of situations were preceded by a white fixation cross (duration from 
(A)

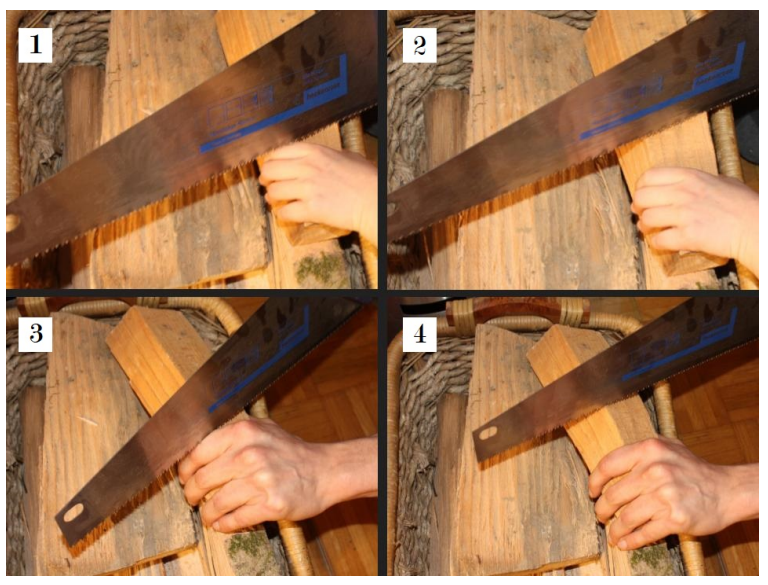

(B)

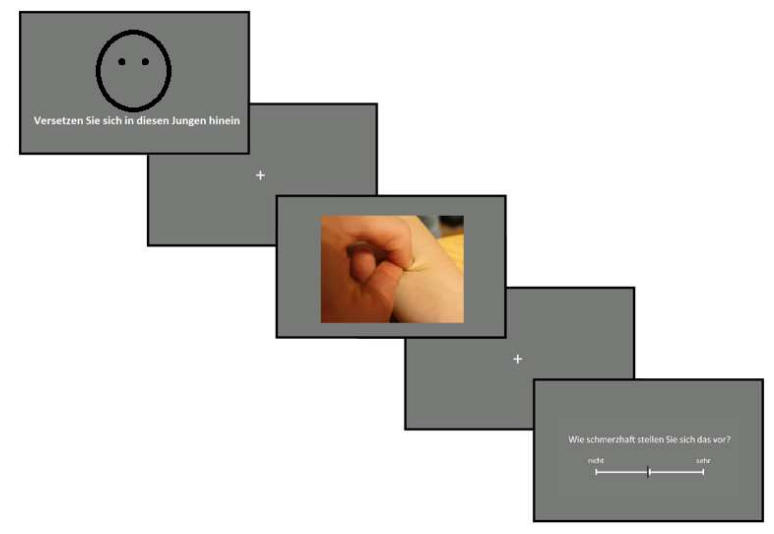

Figure 1: (A) Example for a scenario in all four variations: 1. child painful, 2. child neutral, 3. adult painful, 4. adult neutral. (B) Schematic of the neutral introductory face and the first picture of a trial followed by a rating.

truncated exponential, $\lambda=1.01$, mean $=2.5 \mathrm{~s}$, min $=2.0 \mathrm{~s}$ ). After $50 \%$ of the pictures they were asked to rate how painful they imagine this situation to be on a continuous rating scale ranging from "not" to "very" with two buttons using their index and ring finger of the right hand. Importantly, these ratings are not empathy ratings as they ask to rate the imagined painfulness and not the resulting feeling. Participants had 3s to rate the imagined painfulness without having to confirm their answer. Each rating was separated from the corresponding picture with a fixation cross for an average of 1.3s. Between trials, empty grey screens were presented for 4 to $7 \mathrm{~s}$ (average $4.8 \mathrm{~s}$ ). Pictures were presented in a pseudo-randomised order with the restriction that corresponding painful and neutral situations were not presented close to each other. Additionally, there were never more than two trials of the same protagonist presented after each other.

\subsection{Sample characteristics and imagined painfulness}

All analysis here were performed in JASP (scripts and data: https://osf.io/kyr8v/) [40]. Evidence was labelled as anecdotal, moderate, strong, very strong or decisive based on the adaptation of Jeffrey's scheme used in JASP [41]. Bayesian Mann-Whitney-U tests were computed with 10,000 random samples to compare mothers and non-mothers in age, socio-economic status (SES [42]), intelligence score [31], importance of having children, 
questionnaire scores and mood state. All Mann-Whitney-U tests were corrected for multiple comparisons using Westfall's method [43, 44]. Bayesian contingency tables were used to compare relationship status between mothers and non-mothers. Imagined painfulness during the fMRI paradigm was analysed with a 2x2x2 Bayesian mixed ANOVA with factors motherhood, painfulness and protagonist (child or adult). Variables that showed at least moderate evidence for a group difference in the Bayesian Mann-Whitney-U tests would have been added as covariates to the null model, however, none fulfilled the criterion.

\section{5 fMRI data acquisition}

All scans were acquired using a 20-channel 3 Tesla MRI (Siemens Magnetom Prisma, Siemens Medical Solutions, Erlangen, Germany) at the Berlin Center for Advanced Neuroimaging. Functional images were acquired as T2*-weighted echo-planar images (EPI) across six runs, two of which have been analysed for this paper. Each of the analysed runs consisted of 256 scans acquired in 32 consecutive slices of $3 \mathrm{~mm}$ (voxel size $=3 \mathrm{~mm}^{3}$, $\left.\mathrm{TR}=2000 \mathrm{~ms}, \mathrm{TE}=30 \mathrm{~ms}, \mathrm{FA}=78^{\circ}, \mathrm{FOV}=192 \mathrm{~mm}\right)$. Prior to the functional runs, a structural image was acquired using a T1-weighted magnetically prepared rapid acquisition gradient echo with a $1 \mathrm{~mm}^{3}$ voxel size $(176$ slices, $\mathrm{TR}=2539 \mathrm{~ms}$, $\mathrm{TE}=4.94 \mathrm{~ms}$, $\left.\mathrm{FA}=7^{\circ}, \mathrm{FOV}=256 \mathrm{~mm}\right)$. Between the structural and the functional images, field maps were acquired for each participant (32 slices á 3mm, TR $=400 \mathrm{~ms}$, TE1 $=5.19 \mathrm{~ms}$, TE2 $\left.=7.65 \mathrm{~ms}, \mathrm{FA}=60^{\circ}, \mathrm{FOV}=192 \mathrm{~mm}\right)$.

\section{6 fMRI data preprocessing}

All images were preprocessed using fMRIPrep 20.0.6 [45, 46] which is an automated preprocessing pipeline (for an extensive description of all steps applied by fMRIPrep see the supplementary materials). Anatomical images were corrected for intensity non-uniformity and used as T1 weighted references which were skull-stripped and segmented into tissuetypes. After surface reconstruction, brain masks were refined and spatially normalised to the Montreal Neurological Institute space (MNI152NLin2009cAsym [47]). For each run 
of functional images, susceptibility distortion based on fieldmaps, coregistration, realignment, slice time correction and normalisation were performed. Participants who moved more than one voxel $(3 \mathrm{~mm})$ were excluded from analysis. The preprocessed images from fMRIPrep were subsequently detrended based on a linear model of global signal [48] to remove global effects from the time series. Then, images were continued to be smoothed in SPM12 (Wellcome Department of Imaging Neuroscience, University College London, UK, 2014) using a full width half maximum of $6 \mathrm{~mm}$ of the Gaussian smoothing kernel. All images were masked with participants' individual brain masks.

\section{7 fMRI analysis}

All univariate analyses of the fMRI data were performed using the general linear model as implemented in the SPM12 software. For each subject, one first-level GLM including both runs was specified for the total duration of the pictures in the four conditions shown in table 1B. A "painful > neutral" contrast was computed to measure empathy response separately for adult and child stimuli (scripts and contrasts: https://osf.io/kyr8v/). Group level differences were assessed using a flexible factorial design specification based on the contrast images computed in the first-level analysis. The factors of the model were motherhood (between) and protagonist (within) as well as their interaction. To test the hypotheses, a region-of-interest analysis was performed using a single mask including medial SFG $(-2,24,38)$, left SMG $(-62,-22,32)$ and bilateral AI (left AI; -38, 16, -4; right $\mathrm{AI} ; 44,8,-4)$. Centres are based on peaks from a recent meta-analysis and spheres of $10 \mathrm{~mm}$ were created around each centre to create one mask containing all four spheres [24]. A whole-brain analysis was performed with a cluster-level threshold of family-wise error (FWE) corrected $p<0.05$ to explore additional differences. All contrasts were masked with a $10 \%$ probability grey matter mask provided by SPM12. 
Table 1: Mean, standard error and corrected Bayes factor for all Bayesian Mann-Whitney$\mathrm{U}$ tests performed on answers from the interview and on scores from the questionnaires.

\begin{tabular}{lrrr}
\hline Measurement & \multicolumn{1}{c}{ mothers } & non-mothers & $B F_{10}$ \\
\hline age & $38.3( \pm 0.8)$ & $35.6( \pm 1.4)$ & 0.179 \\
importance of having children $(0-4)$ & $3.7( \pm 0.1)$ & $2.7( \pm 0.3)$ & 0.443 \\
minIQ & $27.7( \pm 2.1)$ & $30.9( \pm 2.2)$ & 0.107 \\
mood state (0-4) & $3.1( \pm 0.1)$ & $33.1( \pm 0.1)$ & 0.810 \\
ECR-RS & $23.9( \pm 1.4)$ & $32.4( \pm 1.8)$ & $1.993+$ \\
ERQ & $41.0( \pm 1.3)$ & $42.4( \pm 1.4)$ & 0.091 \\
IRI-emp & $44.9( \pm 1.1)$ & $43.1( \pm 1.4)$ & 0.119 \\
IRI-PT & $15.3( \pm 0.4)$ & $15.1( \pm 2.2)$ & 0.083 \\
KSE-G & $1.8( \pm 0.1)$ & $2.0( \pm 0.1)$ & 0.403 \\
SES & $14.5( \pm 0.6)$ & $14.0( \pm 0.7)$ & 0.091 \\
TAS & $39.0( \pm 1.8)$ & $40.2( \pm 1.9)$ & 0.081 \\
\hline
\end{tabular}

$\mathrm{ECR}-\mathrm{RS}=$ relationship attachment, $\mathrm{ERQ}=$ emotion regulation quotient, $\mathrm{IRI}=$ interpersonal reactivity, emp = empathy, $\mathrm{PT}=$ perspective taking, $\mathrm{KSE}=$ tendency for social desirability, $\mathrm{SES}=$ socioeconomic score, TAS = alexithymia score; + signifies anecdotal evidence (according to Jeffrey's scheme [40])

\section{Results}

\subsection{Sample characteristics}

Comparisons of questionnaire scores and answers in the interview showed that mothers and non-mothers were comparable in most aspects (see table 1). However, mothers were more likely to be in a relationship than non-mothers with a Bayesian Contingency Table showing decisive evidence in favour of the alternative hypothesis. While only $20 \%$ of mothers were single, $76 \%$ of the non-mothers were single $\left(B F_{10}=975\right)$. Additionally, there was anecdotal evidence that mothers gave higher relationship attachment ratings than non-mothers as measured with the ECR-RS $\left(B F_{10}=1.993\right)$.

\subsection{Imagined painfulness}

The Bayesian mixed ANOVA determined that the model which represents the data best only includes the main factor of imagined painfulness. This model shows decisive evidence in favour of the alternative hypothesis that pictures that were used as painful stimuli were indeed rated higher on the imagined painfulness scale than neutral pictures $\left(B F_{10}=\right.$ $3.3 * 10^{91}$ ). Adding other factors or interactions did not improve the model (see table S1 in the supplementary materials). Inclusive Bayes factors for each factor separately over 


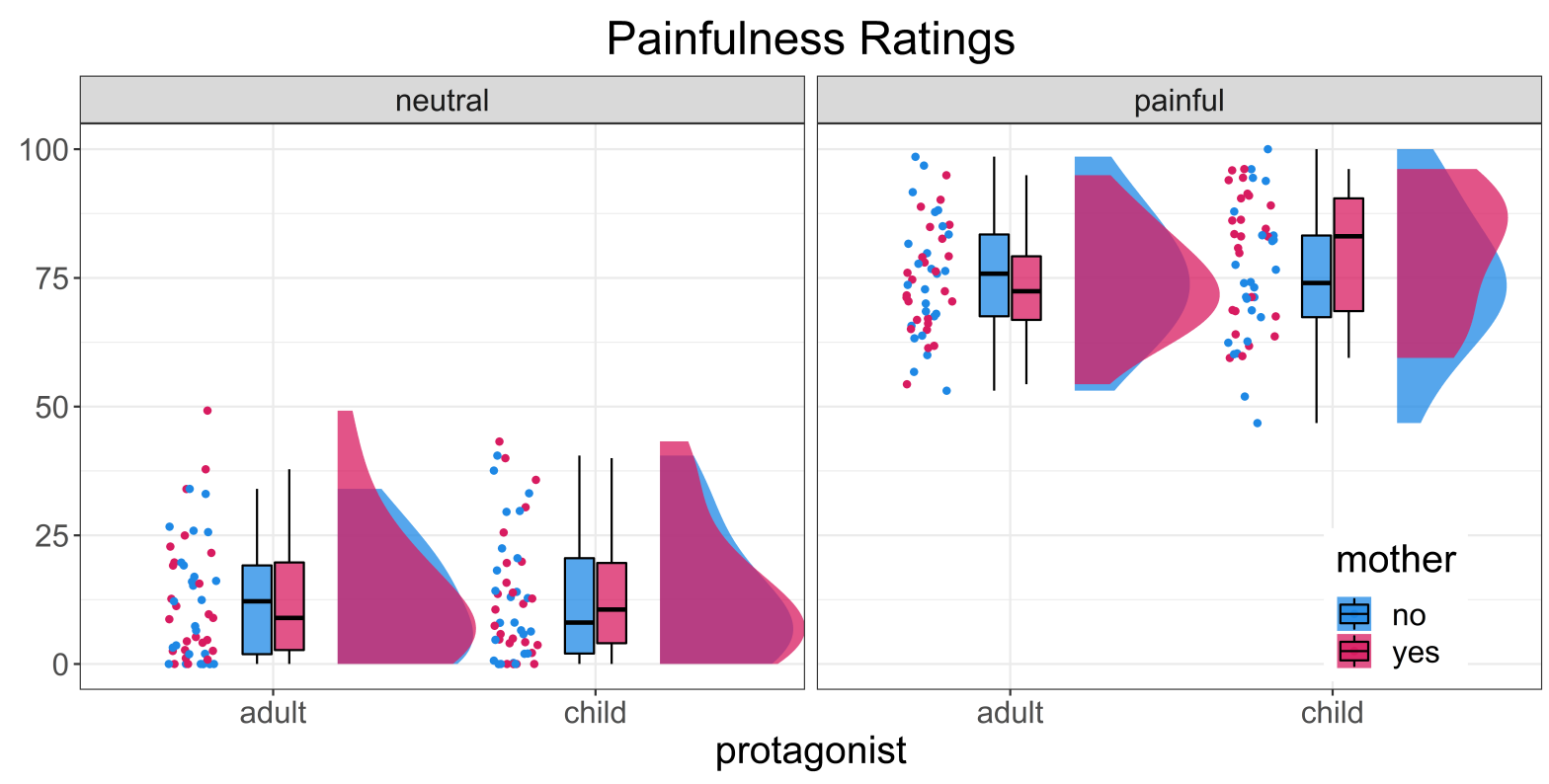

Figure 2: Imagined painfulness rated during the fMRI paradigm. The imagined painfulness of half of all stimuli was rated on a continuous scale from 0 to 100 .

all models shows decisive evidence for the inclusion of pain but moderate evidence against the inclusion of protagonist or mother. Therefore, the experimental manipulation was successful but there were no differences between mothers and non-mothers in how painful a stimulus was imagined and no difference due to the protagonist of the stimulus (i.e., adult or child) or the interaction of both factors (see figure 2).

\section{3 fMRI results}

Both mothers and non-mothers reliably exhibited a strong empathy for pain response (see table S2 and figure S1 in the supplementary materials). The region-of-interest (ROI) analysis confirmed stronger neural responses for mothers relative to non-mothers in the bilateral AI (left: $t=5.59, k_{\mathrm{E}}=124$; right: $t=4.43, k_{\mathrm{E}}=81$ ). There were no activation differences between mothers and non-mothers in the mSFG or ISMG. Adult and child stimuli did not lead to differences in activations in any of the ROIs. There was also no indication for an interaction of both factors in the ROI analysis.

The whole-brain analyses showed additional differences in activation patterns between mothers and non-mothers as well as in responses to child and adult stimuli, however, no interaction between the two (see table 2 and figure 3). Mothers had a higher neural em- 
pathy response than non-mothers in frontal areas, including the bilateral inferior frontal gyrus (IFG) extending into the bilateral insula and right medial SFG, as well as the right superior temporal gyrus (STG) and cuneus, left cerebellum and rolandic operculum (RO). Non-mothers relative to mothers showed a stronger neural empathy response in the left superior parietal gyrus (SPG).

Both non-mothers and mothers had a stronger empathy response to child compared to adult protagonists in the right superior occipital gyrus (SOG) and the left cuneus. For the opposite contrast, both groups showed higher activation in response to adult stimuli in the right inferior occipital gyrus and the left middle occipital gyrus.

\section{Discussion}

This study for the first time shows increased neural empathy responses in mothers compared to non-mothers within the bilateral anterior insula known to represent core regions for empathy for pain. While previous studies have already shown increased emotional response to infants' and prepubescent faces [13] and infants' vocalisations [17], this is the first to extend this to empathy for pain in response to complex situations. Increased empathy responses were found in mothers compared to non-mothers in the bilateral anterior insula which are core regions of empathy for pain [25, 24]. This increased empathy response of mothers was found regardless of mothers and non-mothers rating the imagined painfulness of the scenarios similarly indicating differences in empathy and not perception. Importantly, mothers not only reacted more strongly on a neural level to children in pain but also to adults in pain. There was no interaction between the motherhood of the perceiver and the person getting hurt in the scenario. Therefore, mothers showed a stronger empathy response on the neural level regardless of with whom they were empathising.

Importantly, enhanced neural activation of the AI has also been demonstrated for people in pain or suffering themselves [19]. This indicates a shared network for feeling with someone and feeling in general. AI activation has been found across different modalities 
(A)

$\square$ mothers $>$ non-mothers

$\square$ non-mothers $>$ mothers
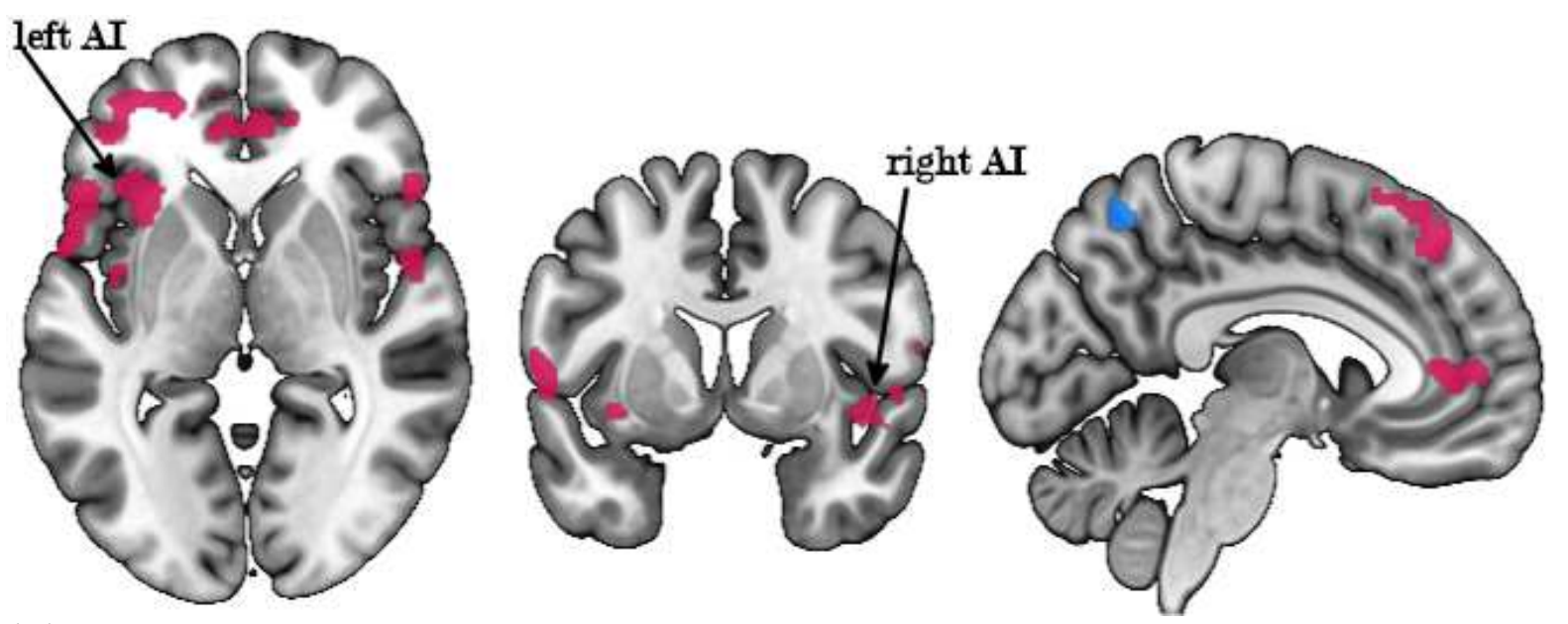

(B)

$\square$ adult $>$ child

$\square$ child $>$ adult
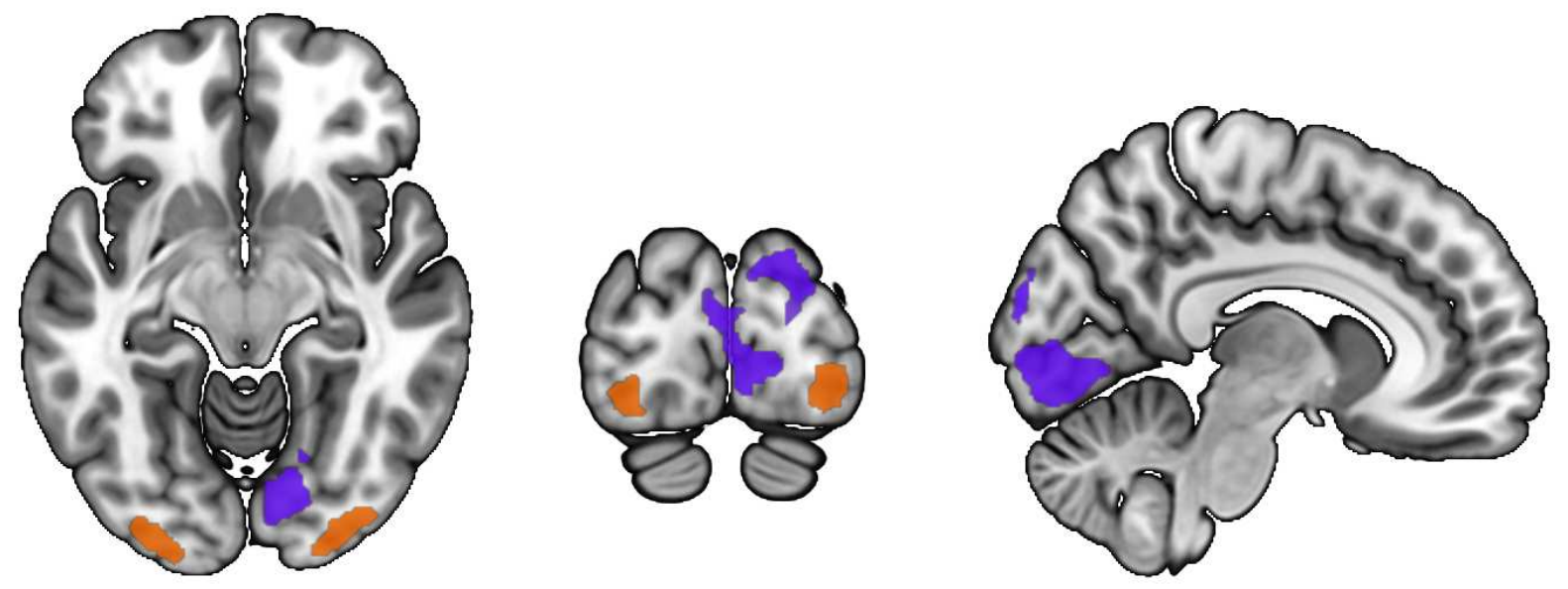

Figure 3: Results of the four main contrasts in the whole-brain analysis with a cluster corrected threshold of $p_{\mathrm{FWE}}<0.05$. Images have been created with MRIcroGL [49]. 
Table 2: Results of the whole-brain analysis based on the flexible factorial model. All contrasts are t-contrasts based on differential first-level contrast images of "pain > neutral" pictures.

\begin{tabular}{|c|c|c|c|c|c|c|c|}
\hline & $\mathrm{BA}$ & $\mathrm{h}$ & $k_{\mathrm{E}}$ & $t$-value & $x$ & $y$ & $z$ \\
\hline \multicolumn{8}{|l|}{ mothers $>$ non-mothers } \\
\hline \multirow{3}{*}{$\begin{array}{l}\text { Inferior frontal gyrus inc. } \\
\text { anterior insula }\end{array}$} & 45 & $\mathrm{~L}$ & 720 & 6.80 & -51 & 22 & -1 \\
\hline & 44 & & & 6.14 & -57 & 8 & 2 \\
\hline & 47 & & & 5.75 & -35 & 26 & -5 \\
\hline \multirow{3}{*}{$\begin{array}{l}\text { Superior temporal gyrus inc. } \\
\text { insula }\end{array}$} & 22 & $\mathrm{R}$ & 1008 & 6.31 & 46 & -33 & 14 \\
\hline & 4 & & & 6.20 & 42 & -5 & 8 \\
\hline & 40 & & & 5.72 & 52 & -17 & 12 \\
\hline \multirow[t]{3}{*}{ Middle frontal gyrus } & 10 & $\mathrm{~L}$ & 380 & 6.26 & -41 & 52 & -5 \\
\hline & 10 & & & 5.18 & -23 & 52 & -3 \\
\hline & 10 & & & 4.71 & -37 & 50 & 10 \\
\hline \multirow[t]{3}{*}{ Cuneus } & 18 & $\mathrm{R}$ & 187 & 6.24 & 22 & -87 & 12 \\
\hline & 18 & & & 4.96 & 18 & -99 & 10 \\
\hline & 18 & & & 4.29 & 16 & -93 & 20 \\
\hline \multirow[t]{3}{*}{ Inferior frontal gyrus } & 45 & $\mathrm{R}$ & 181 & 5.82 & 52 & 24 & 14 \\
\hline & 45 & & & 5.31 & 54 & 26 & -3 \\
\hline & 47 & & & 4.56 & 48 & 24 & -9 \\
\hline \multirow[t]{2}{*}{ Inferior frontal gyrus } & 45 & $\mathrm{~L}$ & 126 & 5.60 & -55 & 24 & 18 \\
\hline & 45 & & & 4.85 & -47 & 24 & 16 \\
\hline \multirow[t]{3}{*}{ Superior frontal gyrus } & 8 & M & 124 & 5.55 & -5 & 38 & 48 \\
\hline & 8 & & & 5.54 & -5 & 34 & 58 \\
\hline & 6 & & & 4.07 & -5 & 22 & 60 \\
\hline \multirow[t]{2}{*}{ Cerebellum (Crus-1) } & & $\mathrm{L}$ & 124 & 5.26 & -23 & -79 & -27 \\
\hline & & & & 4.53 & -35 & -75 & -25 \\
\hline \multirow[t]{3}{*}{ Rolandic operculum } & 4 & $\mathrm{~L}$ & 210 & 5.04 & -43 & -7 & 8 \\
\hline & 1 & & & 4.74 & -39 & -17 & 16 \\
\hline & 13 & & & 4.34 & -35 & -9 & 14 \\
\hline \multirow[t]{3}{*}{ Orbital superior frontal gyrus } & 10 & $\mathrm{R}$ & 242 & 4.64 & 16 & 48 & -5 \\
\hline & 10 & & & 4.61 & 4 & 46 & 2 \\
\hline & 32 & & & 4.50 & -7 & 46 & 2 \\
\hline \multirow[t]{3}{*}{ Superior frontal gyrus } & 9 & $\mathrm{R}$ & 224 & 4.47 & 14 & 54 & 28 \\
\hline & 9 & & & 4.43 & 22 & 50 & 28 \\
\hline & 10 & & & 4.22 & 16 & 62 & 22 \\
\hline \multicolumn{8}{|l|}{ non-mothers $>$ mothers } \\
\hline \multirow[t]{3}{*}{ Superior parietal gyrus } & 7 & $\mathrm{~L}$ & 320 & 5.86 & -23 & -63 & 62 \\
\hline & 7 & & & 5.60 & -9 & -63 & 58 \\
\hline & 7 & & & 5.45 & -37 & -63 & 58 \\
\hline \multicolumn{8}{|l|}{ adults $>$ children } \\
\hline Inferior occipital gyrus & 18 & $\mathrm{R}$ & 239 & 5.97 & 36 & -87 & -9 \\
\hline Middle occipital gyrus & 18 & $\mathrm{~L}$ & 148 & 5.54 & -27 & -99 & -7 \\
\hline \multicolumn{8}{|l|}{ children $>$ adults } \\
\hline \multirow[t]{3}{*}{ Superior occipital gyrus } & 19 & $\mathrm{R}$ & 1020 & 6.13 & 22 & -91 & 30 \\
\hline & 18 & & & 5.95 & 12 & -81 & -11 \\
\hline & 17 & & & 5.02 & 8 & -91 & -1 \\
\hline \multirow[t]{2}{*}{ Cuneus } & 18 & $\mathrm{~L}$ & 123 & 4.89 & -11 & -77 & 30 \\
\hline & 7 & & & 4.08 & -17 & -67 & 32 \\
\hline
\end{tabular}

Cluster-corrected $p_{\mathrm{FWE}}<0.05$; all results are grey matter masked; $\mathrm{L}=$ left, $\mathrm{R}=$ right, $\mathrm{M}=$ medial, $k_{\mathrm{E}}=$ cluster size, coordinates are in MNI space and are the location of the peak voxel for each cluster. 
suggesting that the AI is encoding the emotional effect and not the sensory features [50]. Studies have found that activation in the AI correlates with empathy ratings [51]. Higher activity in the AI also predicted higher motivation to help a person in pain [52]. Both suggests that higher activity translates to higher empathy. Therefore, the higher neural empathy response in mothers found in this study might indicate that mothers felt more empathy than non-mothers.

These effects in the neural empathy response were found despite no differences in imagined painfulness between mothers and non-mothers. This indicates that even though mothers and non-mothers assessed the physical pain the same, their reaction was different. If mothers and non-mothers had rated the imagined painfulness of the stimuli differently, their difference in interpretation of the imagined painfulness might have been the driving force. However, since there were no significant differences between the groups in the imagined painfulness of the stimuli, this indicates that faced with the same scenarios, mothers and non-mothers interpret the situation similarly but then react differently to it with mothers exhibiting a stronger empathy response.

Additionally to a higher empathy response in empathy for pain regions, mothers' empathy response was also characterised by increased activation in other regions associated with social understanding compared to non-mothers' empathy response. This included regions that a recent hierarchical analysis of social understanding associated with affective processes, like the triangular part of the IFG and the right STG, both extending into the insula. These areas have been linked to shared networks that are activated both when observing for example an emotional facial expression and producing it oneself [5]. This shared activation is assumed to facilitate understanding of others [53, 54]. These results combined with our findings further consolidate the interpretation that mothers' affective reaction to the same stimuli might have been stronger than the reaction of non-mothers.

Furthermore, mothers showed higher activation in their empathy response in regions commonly associated with predominantly cognitive processes of social understanding like the SFG, RO [5] and the cerebellum [55]. Cognitive processes might have been more strongly involved due to the complexity and naturalism of the stimuli. The close matching 
between painful and neutral pictures has the benefit that differences are due to the difference in empathy response and not reactions to threatening stimuli. However, it also meant that participants had to closely pay attention to interpret the given scenario correctly. For example, some pictures contained needles. While in the neutral version, the needle was wearing a cap, it was still held close to the skin. Participants had to see the cap and infer that due to the needle being capped this contact between skin and needle is not painful. The differences in activation in these areas between mothers and non-mothers might indicate that additionally to stronger feeling with the people in the scenarios, mothers also employed additional cognitive resources to interpret the stimuli and possibly understand the scenarios and the people in them better.

This study cannot determine the causal relationship between motherhood and empathy response. On the one hand, the differences in empathy response could be due to the experiences of motherhood itself: the intense process of caring for another human being means that mothers increasingly rely on their empathy system. Therefore, the connections are strengthened and they exhibit a stronger response to the same scenarios. Several studies have shown that empathy can be increased by training [56, 57]. Motherhood could have similar training effects simply due to everyday life situations with their children. On the other hand, it is possible that women with a stronger empathy response are more likely to become mothers. Additional research is needed to disentangle these options from one another.

Surprisingly, empathy responses in brain regions of interest were not modulated by the protagonist of the stimulus in women. All differences between adults and child stimuli were confined to the occipital lobe in areas most commonly associated with visual processing. This means that it is likely that women reacted with the same amount of empathy to watching children and adults in pain. Additionally, mothers also did not react more strongly to children than non-mothers did. Both missing effects might be due to the stimulus material: in an effort to keep adult and child stimuli comparable, only body parts and not faces were shown in the scenarios and pictures were closely matched. As a consequence, the difference between child and adult scenarios were in some cases 
very subtle. We used faces to introduce the scenarios to explicitly point out the protagonist of the following scenarios but the differences still might have been too subtle in our paradigm. Previous studies have shown that the empathy for pain paradigm employed here is sensitive to at least some differences due to protagonist, for example race [22]. However, most studies have used less complex stimuli and therefore participants might have focussed more on the protagonist while in this paradigm they could have focussed on the overall situation. Moreover, in most studies, out-group protagonists led to a diminished or reduced empathy response. In our study, adult protagonists were the in-group but we expected increased empathy response to child stimuli. It is possible that children's special status leads to them being treated as in-group instead of out-group but not to an additional increase in empathy response. While empathy responses in empathy areas did not differ between protagonists, differences might still surface when focussing on the consequences of the empathy response: children in pain could lead to more compassion and a stronger motivation to help. Our empathy for pain paradigm was designed to elicit empathy by asking participants to imagine themselves in the scenarios. Although empathy can lead to compassion, compassion was not encouraged in this paradigm. Last but not least, higher empathy responses towards children could be strictly confined to the own offspring [58]. Therefore, there are several possibilities that could explain why empathy response in empathy areas was not influenced by the protagonist in our paradigm. Further research is needed to investigate and disentangle these possibilities.

The effect of motherhood also should be subject of further scientific investigation. This study compared biological mothers to non-mothers who do not have close and/or frequent contact with children. This implied three factors that might operate independently of each other: biological parenthood, motherhood and contact with children. First, studies have found many similarities between adoptive and biological mothers, both in attention allocation [59] and emotional reaction functions [60]. A study comparing biological and adoptive mothers who adopted their children as infants might find effects of motherhood on empathy similar to those found in the present study. Second, recent studies have started to investigate the influence that fatherhood has on men and how this compares 
to the influence of motherhood to women [29]. Therefore, it would be interesting to see if our results can be replicated in a sample of primary-care fathers. Third, apart from parents there are also people who made childcare their profession. Investigating kindergarteners, nannies and similar professionals who are not parents could answer the following questions: is it the responsibility for and care of children that leads to an increased empathy response? Or is it the special bond between parents and their children that fosters these differences? More research is needed to disentangle these factors and shed further light on the connection between parenthood, childcare and empathy.

This study offers the first glimpse in generalisable differences in empathy between mothers and non-mothers. Using an empathy for pain paradigm, women were confronted with matched painful and neutral pictures depicting children or adults as protagonists. Empathy response was computed by subtracting neural responses to the neutral pictures from the painful pictures. Mothers exhibited a higher empathy response than non-mothers in core regions of empathy as well as cognitive social understanding despite both groups giving the same ratings for imagined painfulness of the pictures. Surprisingly, whether the protagonist of the stimulus was a child or an adult did not alter the empathy response outside of visual processing areas. There was also no interaction between the protagonist of the stimuli and motherhood. Therefore, mothers in this study showed a higher general empathy response than non-mothers regardless of the target of their empathy.

\subsection{Data availability}

Data and scripts to reproduce the results are available in the Open Science Framework repository: https://osf.io/kyr8v/. 


\section{References}

[1] Batson, C. D., Fultz, J. \& Schoenrade, P. A. Distress and Empathy: Two Qualitatively Distinct Vicarious Emotions with Different Motivational Consequences. Journal of Personality 55, 19-39 (1987).

[2] de Vignemont, F. \& Singer, T. The empathic brain: how, when and why? Trends in Cognitive Sciences 10, 435-441 (2006).

[3] Kanske, P., Böckler, A., Trautwein, F. M., Lesemann, F. H. \& Singer, T. Are strong empathizers better mentalizers? Evidence for independence and interaction between the routes of social cognition. Social Cognitive and Affective Neuroscience 11, 1383-1392 (2016).

[4] Winter, K., Spengler, S., Bermpohl, F., Singer, T. \& Kanske, P. Social cognition in aggressive offenders: Impaired empathy, but intact theory of mind. Scientific reports 7, 1-10 (2017).

[5] Schurz, M. et al. Toward a Hierarchical Model of Social Cognition: A Neuroimaging Meta-Analysis and Integrative Review of Empathy and Theory of Mind. Psychological Bulletin (2020).

[6] Decety, J., Norman, G. J., Berntson, G. G. \& Cacioppo, J. T. A neurobehavioral evolutionary perspective on the mechanisms underlying empathy. Progress in Neurobiology $\mathbf{9 8}, 38-48$ (2012).

[7] Stern, J. A., Borelli, J. L. \& Smiley, P. A. Assessing parental empathy: a role for empathy in child attachment. Attachment and Human Development 17, 1-22 (2014).

[8] Kluczniok, D. et al. Transgenerational effects of maternal depression on affect recognition in children. Journal of Affective Disorders 189, 233-239 (2016).

[9] Dittrich, K. et al. Alterations of empathy in mothers with a history of early life maltreatment, depression, and borderline personality disorder and their effects on child psychopathology. Psychological medicine 50, 1182-1190 (2020). 
[10] Shinohara, I. \& Moriguchi, Y. Adults' Theory of Infants' Mind: A Comparison between Parents and Nonparents. Child Development Research (2017).

[11] Parsons, C. E., Young, K. S., Jegindoe Elmholdt, E. M., Stein, A. \& Kringelbach, M. L. Interpreting infant emotional expressions: Parenthood has differential effects on men and women. Quarterly Journal of Experimental Psychology 70, 554-564 (2016).

[12] Thompson-Booth, C. et al. Here's looking at you, kid: Attention to infant emotional faces in mothers and non-mothers. Developmental Science 17, 35-46 (2014).

[13] Thompson-Booth, C. et al. I can't take my eyes off of you: Attentional allocation to infant, child, adolescent and adult faces in mothers and non-mothers. PLoS ONE 9, e109362 (2014).

[14] Proverbio, A. M., Brignone, V., Matarazzo, S., Del Zotto, M. \& Zani, A. Gender and parental status affect the visual cortical response to infant facial expression. Neuropsychologia 44, 2987-2999 (2006).

[15] Hayashi, S. et al. Enhanced Nogo-P3 amplitudes of mothers compared with nonmother women during an emotional Go/Nogo task. Journal of Physiological Anthropology $\mathbf{3 7}, 1-8$ (2018).

[16] Nishitani, S., Doi, H., Koyama, A. \& Shinohara, K. Differential prefrontal response to infant facial emotions in mothers compared with non-mothers. Neuroscience Research 70, 183-188 (2011).

[17] Parsons, C. E. et al. Duration of motherhood has incremental effects on mothers' neural processing of infant vocal cues: A neuroimaging study of women. Scientific Reports 7, 1-9 (2017).

[18] Zhang, K. et al. Brain responses to emotional infant faces in new mothers and nulliparous women. Scientific reports 10, 1-10 (2020). 
[19] Singer, T. et al. Empathy for Pain Involves the Affective but not Sensory Components of Pain. Science 303, 1157-1162 (2004).

[20] Cheng, Y. et al. Expertise Modulates the Perception of Pain in Others. Current Biology 17, 1708-1713 (2007).

[21] Singer, T. et al. Empathic neural responses are modulated by the perceived fairness of others. Nature 439, 466-469 (2006).

[22] Han, S. Neurocognitive Basis of Racial Ingroup Bias in Empathy. Trends in Cognitive Sciences 22, 400-421 (2018).

[23] Lamm, C., Meltzoff, A. N. \& Decety, J. How do we empathize with someone who is not like us? A functional magnetic resonance imaging study. Journal of Cognitive Neuroscience 22, 362-376 (2010).

[24] Kogler, L., Müller, V. I., Werminghausen, E., Eickhoff, S. B. \& Derntl, B. Do I feel or do I know? Neuroimaging meta-analyses on the multiple facets of empathy. Cortex (2020).

[25] Lamm, C., Decety, J. \& Singer, T. Meta-analytic evidence for common and distinct neural networks associated with directly experienced pain and empathy for pain. NeuroImage 54, 2492-2502 (2011).

[26] Timmers, I. et al. Is empathy for pain unique in its neural correlates? A metaanalysis of neuroimaging studies of empathy. Frontiers in Behavioral Neuroscience 12, 1-12 (2018).

[27] Bundesministerium für Familie, F. u. J., Senioren. Gender care gap — ein indikator für die gleichstellung. https://www.bmfsfj.de/bmfsfj/themen/ gleichstellung/gender-care-gap/indikator-fuer-die-gleichstellung/ gender-care-gap---ein-indikator-fuer-die-gleichstellung/137294 (2019).

Accessed: 2020-12-16. 
[28] Bundesamt, S. Datenreport $2018 \quad$ - sozialbericht für deutschland. https://www.destatis.de/DE/Service/Statistik-Campus/Datenreport/ Downloads/datenreport-2018.html (2018). Accessed: 2020-12-16.

[29] Abraham, E. et al. Father's brain is sensitive to childcare experiences. Proceedings of the National Academy of Sciences of the United States of America 111, 9792-9797 (2014).

[30] Faul, F., Erdfelder, E., Lang, A.-G. \& Buchner, A. G*Power 3: A flexible statistical power analysis program for the social, behavioral, and biomedical sciences. Behavior research methods 39, 175-191 (2007).

[31] Baudson, T. G. \& Preckel, F. Mini-q: Intelligenzscreening in drei Minuten. Diagnostica 62, 182-197 (2016).

[32] Abler, B. \& Kessler, H. Emotion Regulation Questionnaire - Eine Deutschsprachige Fassung des ERQ von Gross und John. Diagnostica 55, 144-152 (2009).

[33] Fraley, R. C., Heffernan, M. E., Vicary, A. M. \& Brumbaugh, C. C. The Experiences in Close Relationships-Relationship Structures Questionnaire: A Method for Assessing Attachment Orientations Across Relationships. Psychological Assessment 23, 615-625 (2011).

[34] Kemper, C., Beierlein, C., Bensch, D., Kovaleva, A. \& Rammstedt, B. Eine Kurzskala zur Erfassung des Gamma-Faktors sozial erwünschten Antwortverhaltens: Die Kurzskala Soziale Erwünschtheit-Gamma (KSE-G). GESIS - Working Papers 25 (2012).

[35] Popp, K. et al. Faktorstruktur und reliabilität der toronto-alexithymie-skala (tas-20) in der deutschen bevölkerung. PPmP-Psychotherapie. Psychosomatik. Medizinische Psychologie 58, 208-214 (2008).

[36] Paulus, C. Der Saarbrücker Persönlichkeitsfragebogen SPF (IRI) zur Messung von Empathie: Psychometrische Evaluation der deutschen Version des Interpersonal Reactivity Index (2009). URL http://hdl.handle.net/20.500.11780/3343. 
[37] Lundquist, D., Flykt, A. \& Öhman, A. The karolinska directed emotional faces - kdef, cd rom from department of clinical neuroscience, psychology section, karolinska institute (1998).

[38] LoBue, V. \& Thrasher, C. The Child Affective Facial Expression (CAFE) set: Validity and reliability from untrained adults. Frontiers in Psychology 5, 1-8 (2015).

[39] LoBue, V. \& Thrasher, C. The child affective facial expression (café) set. Databrary (2014).

[40] JASP Team. JASP (Version 0.14)[Computer software] (2020). URL https: //jasp-stats.org/.

[41] Goss-Sampson, M. A. Bayesian inference in jasp: A guide for students (2020).

[42] Lampert, T., Kroll, L. E., Müters, S. \& Stolzenberg, H. Messung des sozioökonomischen Status in der Studie zur Gesundheit Erwachsener in Deutschland (DEGS1). Bundesgesundheitsblatt - Gesundheitsforschung - Gesundheitsschutz 56, 631-636 (2013).

[43] Westfall, P. H., Johnson, W. O. \& Utts, J. M. A bayesian perspective on the bonferroni adjustment. Biometrika 84, 419-427 (1997).

[44] de Jong, T. A bayesian approach to the correction for multiplicity. PsyArXiv (2019).

[45] Esteban, O. et al. fMRIPrep: a robust preprocessing pipeline for functional MRI. Nature Methods (2018).

[46] Esteban, O. et al. fmriprep. Software (2018).

[47] Fonov, V., Evans, A., McKinstry, R., Almli, C. \& Collins, D. Unbiased nonlinear average age-appropriate brain templates from birth to adulthood. NeuroImage $\mathbf{4 7}$, Supplement 1, S102 (2009).

[48] Macey, P. M., Macey, K. E., Kumar, R. \& Harper, R. M. A method for removal of global effects from fMRI time series. NeuroImage 22, 360-366 (2004). 
[49] Rorden, C. \& Brett, M. Stereotaxic display of brain lesions. Behavioural neurology 12, 191-200 (2000).

[50] Corradi-Dell'Acqua, C., Tusche, A., Vuilleumier, P. \& Singer, T. Cross-modal representations of first-hand and vicarious pain, disgust and fairness in insular and cingulate cortex. Nature communications 7, 1-12 (2016).

[51] Kanske, P., Böckler, A., Trautwein, F. M. \& Singer, T. Dissecting the social brain: Introducing the EmpaToM to reveal distinct neural networks and brain-behavior relations for empathy and Theory of Mind. NeuroImage 122, 6-19 (2015).

[52] Hein, G., Silani, G., Preuschoff, K., Batson, C. D. \& Singer, T. Neural responses to ingroup and outgroup members' suffering predict individual differences in costly helping. Neuron 68, 149-160 (2010).

[53] Gallese, V., Keysers, C. \& Rizzolatti, G. A unifying view of the basis of social cognition. Trends in Cognitive Sciences 8, 396-403 (2004).

[54] de Waal, F. B. \& Preston, S. D. Mammalian empathy: behavioural manifestations and neural basis. Nature Reviews Neuroscience 18, 498 (2017).

[55] Morelli, S. A. \& Lieberman, M. D. The role of automaticity and attention in neural processes underlying empathy for happiness, sadness, and anxiety. Frontiers in Human Neuroscience 7, 1-15 (2013).

[56] Hildebrandt, L. K., McCall, C. \& Singer, T. Socioaffective versus sociocognitive mental trainings differentially affect emotion regulation strategies. Emotion 19, 1329-1342 (2019).

[57] Trautwein, F. M., Kanske, P., Böckler, A. \& Singer, T. Differential benefits of mental training types for attention, compassion, and theory of mind. Cognition 194 (2020).

[58] Rigo, P. et al. Specific maternal brain responses to their own child's face: An fMRI meta-analysis. Developmental Review 51, 58-69 (2019). 
[59] Grasso, D. J., Moser, J. S., Dozier, M. \& Simons, R. ERP correlates of attention allocation in mothers processing faces of their children. Biological Psychology 81, 95-102 (2009).

[60] Hernández-González, M., Hidalgo-Aguirre, R. M., Guevara, M. A., Pérez-Hernández, M. \& Amezcua-Gutiérrez, C. Observing videos of a baby crying or smiling induces similar, but not identical, electroencephalographic responses in biological and adoptive mothers. Infant Behavior and Development 42, 1-10 (2016).

\section{Acknowledgements}

This work was supported by the German Federal Ministry of Education and Research (BMBF; grant 01KR1803C; https://www.ubica.site) and by the "Dissertation Plus" program of the Claussen-Simon-Stiftung.

\section{Author contributions}

IP, CH, ID and FB conceptualised and designed the study. IP took the lead on acquisition and analysis with contributions of $\mathrm{CH}$. IP wrote the draft. All authors contributed to the interpretation of the data and the revision of the manuscript. All authors provided critical feedback and helped shape the research, analysis and manuscript.

\section{Competing interests}

The authors declare no competing interests. 


\section{Figures}

(A)

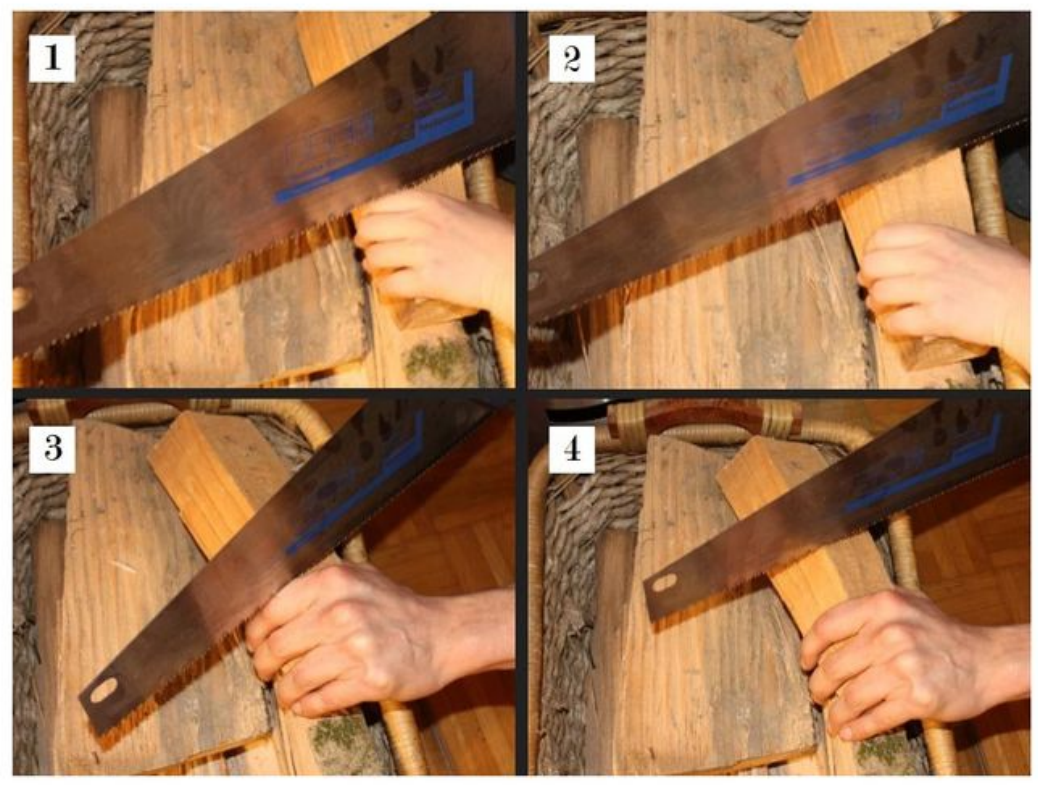

(B)

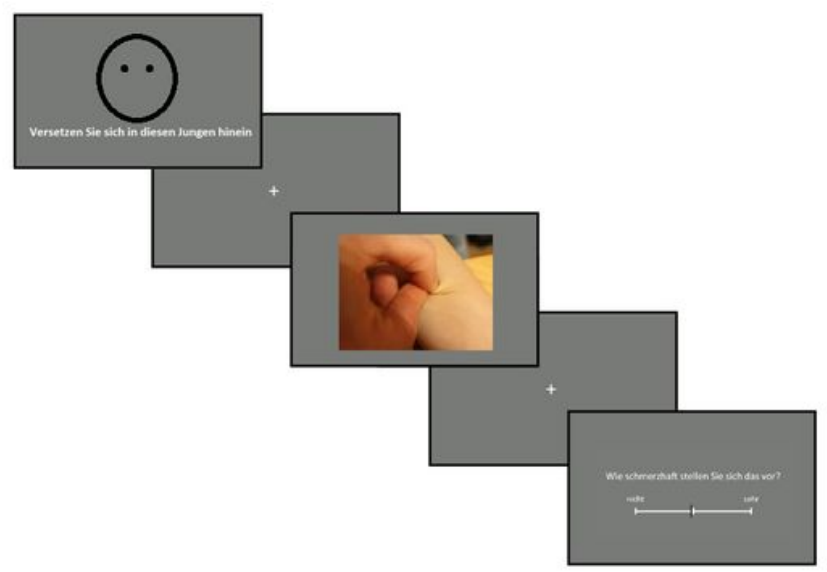

\section{Figure 1}

(A) Example for a scenario in all four variations: 1. child painful, 2. child neutral, 3. adult painful, 4. adult neutral. (B) Schematic of the neutral introductory face and the rst picture of a trial followed by a rating.

\section{Painfulness Ratings}

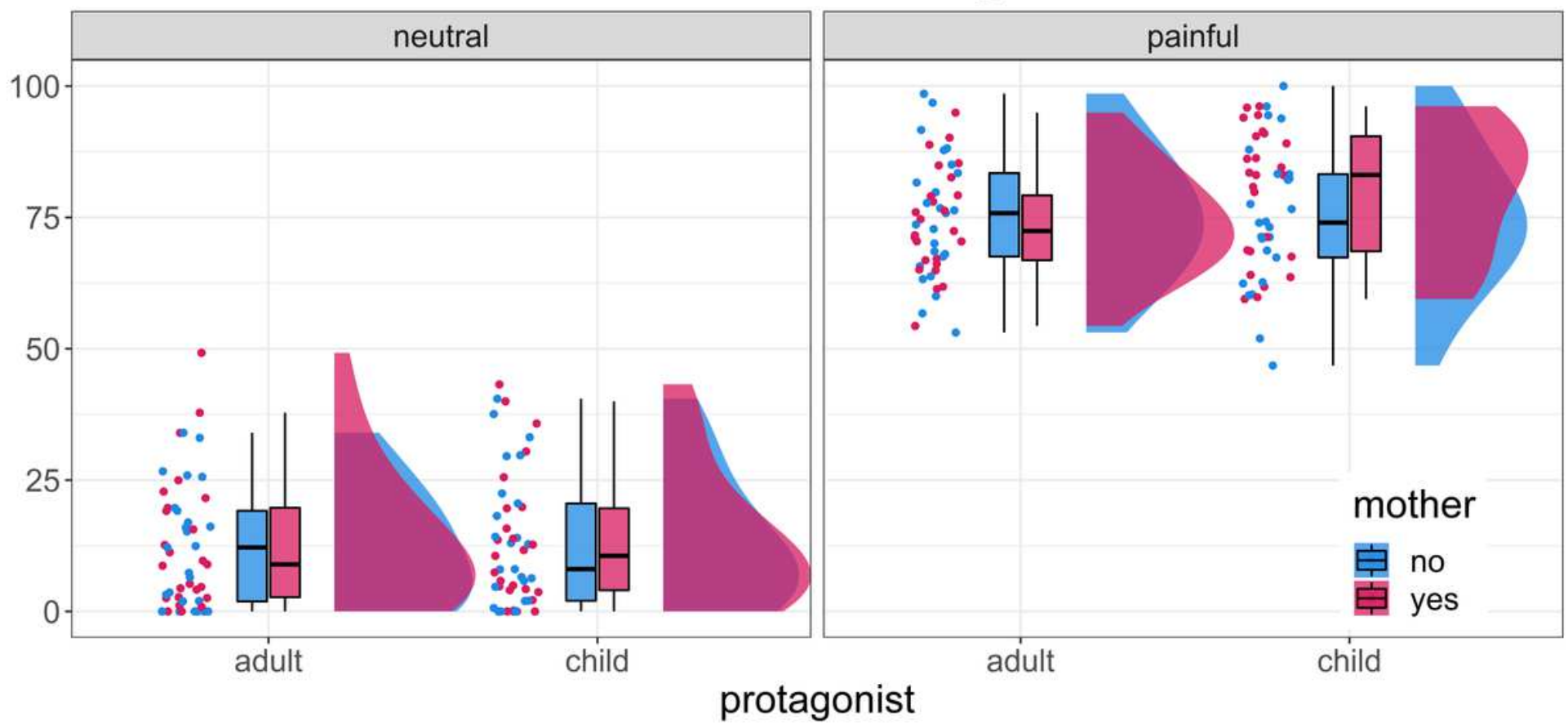

Figure 2 
Imagined painfulness rated during the fMRI paradigm. The imagined painfulness of half of all stimuli was rated on a continuous scale from 0 to 100 .

(A)

$\square$ mothers > non-mothers

$\square$ non-mothers > mothers
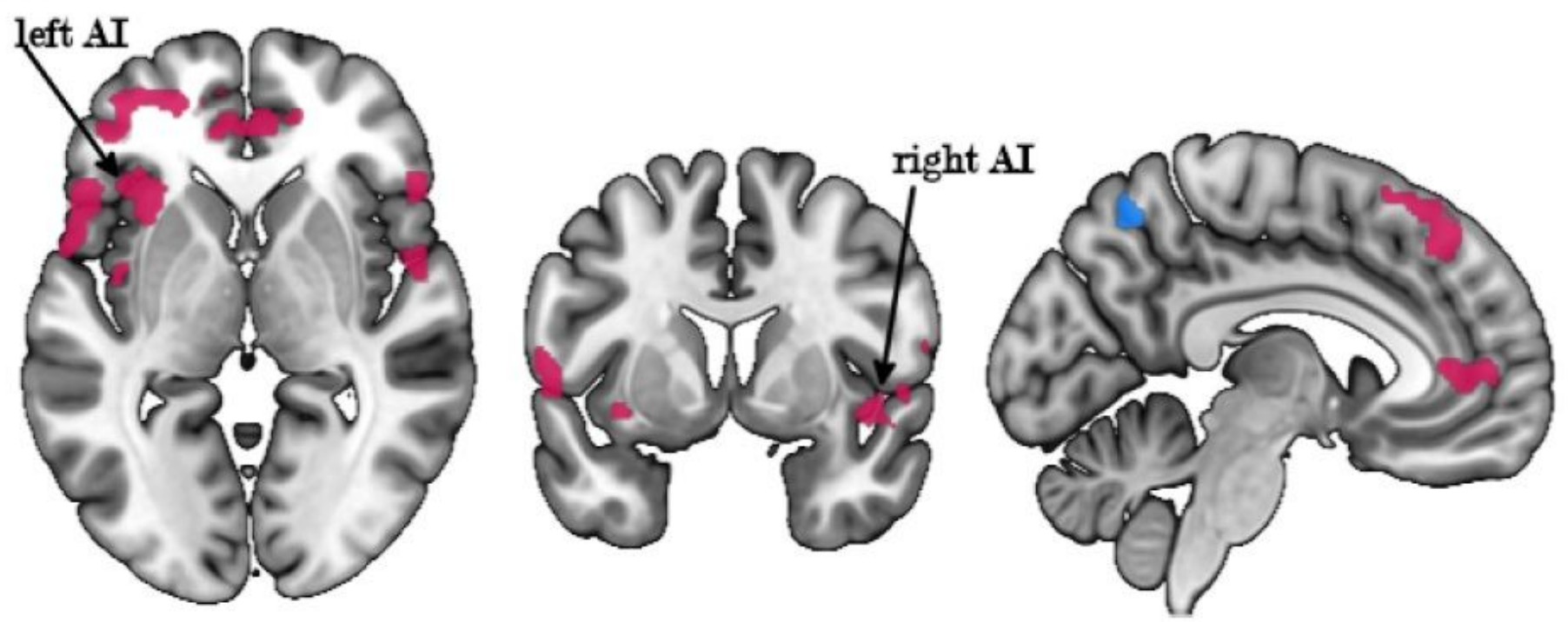

(B)

$\square$ adult $>$ child

$\square$ child $>$ adult
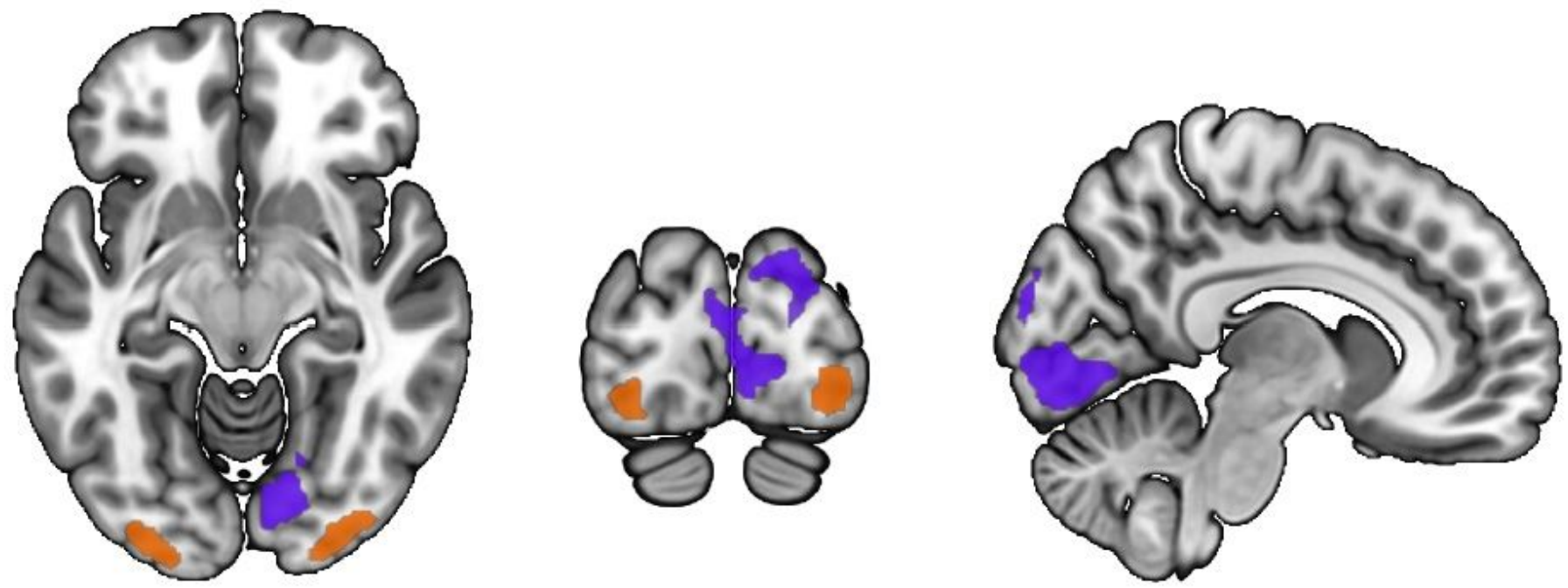

Figure 3

Results of the four main contrasts in the whole-brain analysis with a cluster corrected threshold of $\mathrm{pFWE}$ $<0: 05$. Images have been created with MRIcroGL [49].

\section{Supplementary Files}


This is a list of supplementary files associated with this preprint. Click to download.

- painmotherssup.pdf 